\title{
Fatal interstitial lung disease associated with Crizotinib pathologically confirmed by percutaneous lung biopsy in a patient with ROS1-rearranged advanced non-small-cell lung cancer: a case report
}

\author{
Shibo $\mathrm{Wu}^{1+} \mathbb{D}$, Kaitai Liu ${ }^{2+}$, Feng Ren ${ }^{3}$, Dawei Zheng ${ }^{4}$ and Deng Pan ${ }^{5^{*}}$
}

\begin{abstract}
Background: Crizotinib is a multi-target inhibitor approved for the treatment of advanced non-small-cell lung cancer patients with a ROS1 rearrangement. However, interstitial lung disease is a rare but severe and fatal side effect of crizotinib that should lead to immediate discontinuation of the drug. Unfortunately, the pathophysiology, molecular mechanism and risk factors for crizotinib-induced interstitial lung disease remain poorly understood.

Case presentation: We first identified and reported interstitial lung disease induced de novo by crizotinib in a 47-year-old female patient who was diagnosed with advanced lung adenocarcinoma with a ROS1 rearrangement in a malignant pleural effusion. Subsequent next-generation sequencing analysis revealed both ROS1 rearrangement and an EGFR exon 19 deletion mutation in lung biopsy specimens, which were histologically confirmed to be interstitial lung disease. Although crizotinib treatment was ceased immediately and a shock treatment with high-dose methylprednisolone as well as other necessary treatment procedures was applied to reverse the interstitial lung disease process, the patient died.

Conclusions: The present case indicates that while treating non-small-cell lung cancer patients with crizotinib, it is important to constantly monitor any newly emerging respiratory symptoms and unexplained imaging changes, which may suggest an adverse effect related to drug-induced interstitial lung disease or even lethality. Histopathology and molecular pathological examination of lung biopsy specimens may help clinicians understand the development mechanism and exclude other causes.
\end{abstract}

Keywords: Interstitial lung disease, Crizotinib, ROS1 rearrangement, EGFR mutation

\section{Background}

Crizotinib is a multi-target inhibitor, which was granted a full approval by the Food and Drug Administration (FDA) for the treatment of advanced non-small-cell lung cancer (NSCLC) patients with a ROS1 rearrangement in March 2016. However, interstitial lung disease (ILD) is a rare but severe and fatal side effect of crizotinib that should lead to immediate discontinuation of the drug.

\footnotetext{
*Correspondence: pand2008@yeah.net; 178042519@qq.com

+Shibo Wu and Kaitai Liu contributed equally to this work.

${ }^{5}$ Department of Diagnosis, Ningbo Diagnostic Pathology Center, No. 79 ,

Huan'cheng Road, Ningbo 315021, China

Full list of author information is available at the end of the article
}

Unfortunately, the pathophysiology, molecular mechanism and risk factors for crizotinib-induced ILD remain poorly understood. Here, we describe a case of SDC4-ROS1 rearrangement-positive advanced lung adenocarcinoma with de novo crizotinib-induced ILD.

\section{Case presentation}

A 47-year-old female Chinese patient was admitted to our hospital in January 2018 due to complaints of continuous cough and a feeling of breathlessness for more than a week. The patient did not have a history of alcohol consumption or smoking. She refused to reveal a

(c) The Author(s). 2018 Open Access This article is distributed under the terms of the Creative Commons Attribution 4.0 International License (http://creativecommons.org/licenses/by/4.0/) which permits unrestricted use, distribution, and 
special occupational history and the medical history of her family.

A chest computed tomography (CT) scan revealed a large, irregularly shaped mass on the upper right lobe, accompanied by multiple nodules, plaques and consolidated masses of different sizes, randomly distributed in both lung fields. Nodular thickening of the interlobular septa and fissures, which suggested lymphangitis carcinomatosa, hilar and mediastinal lymphadenopathy and bilateral pleural effusions, was identified by the CT scan as well (Fig. 1a).

An immediate drainage was conducted for the right pleural effusion, followed by a series of tests. Methylprednisolone (MP) at $80 \mathrm{mg} /$ day was administered to alleviate dyspnoea associated with lymphangitis carcinomatosa. With oxygen therapy via a nasal catheter at a flow rate of $6 \mathrm{~L} / \mathrm{min}$, her arterial blood gas was measured to have values of a $\mathrm{PaO}_{2}$ of $55.0 \mathrm{mmHg}$, a $\mathrm{PaCO}_{2}$ of $32.0 \mathrm{mmHg}$, and a $\mathrm{pH}$ of 7.49. The carcinoembryonic antigen (CEA) level in hydrothorax was $7.5 \mu \mathrm{g} / \mathrm{L}$ (normal $0-5 \mu \mathrm{g} / \mathrm{L}$ ), whereas the serum CEA level was $12.4 \mu \mathrm{g} / \mathrm{L}$. The rest of the important blood and sputum test indicators are described in Table 1.

With a poor performance status $(\mathrm{PS}=4)$, the patient was unable to withstand tissue biopsy acquisition. A great number of tumour cells positive for thyroid transcription factor-1 (TTF-1) and cytokeratin 7 (CK 7) were confirmed by pathological haematoxylin-eosin (HE) staining examination of hydrothorax, combined with immunohistochemical staining. These observations led to a diagnosis of advanced lung adenocarcinoma with extensive dissemination in the chest (Fig. 2a-c).

Next-generation sequencing was then conducted on tumour cells of hydrothorax. The SDC4-ROS1 fusion gene was detected at an abundance of $19.8 \%$ in the malignant pleural effusion (MPE). Other mutations, such as those in the EGFR, ALK, KRAS, BFAF, HER2, PIK3CA, MET, and RET genes, were not detected (Fig. 3 Z17L06517, Fig. 4b). The patient was thus orally administered crizotinib at a dose of $250 \mathrm{mg}$ twice per day. After three days of crizotinib treatment, the orthopnoea was greatly relieved, and MP medication was withdrawn. However, oxygen therapy was still required within a one-week time frame of administration. On the tenth day of medication, the patient had a low-grade fever and slight aggravation of dyspnoea. A chest CT re-scan revealed a significant shrinkage of intrapulmonary neoplastic lesions, lymphadenitis and lymphadenectasis. However, multiple new-onset ground-glass opacities and consolidations were detected throughout both lungs (Fig. 1b). The patient was then additionally treated with cefoperazone/sulbactam to exclude the possibility of infection.
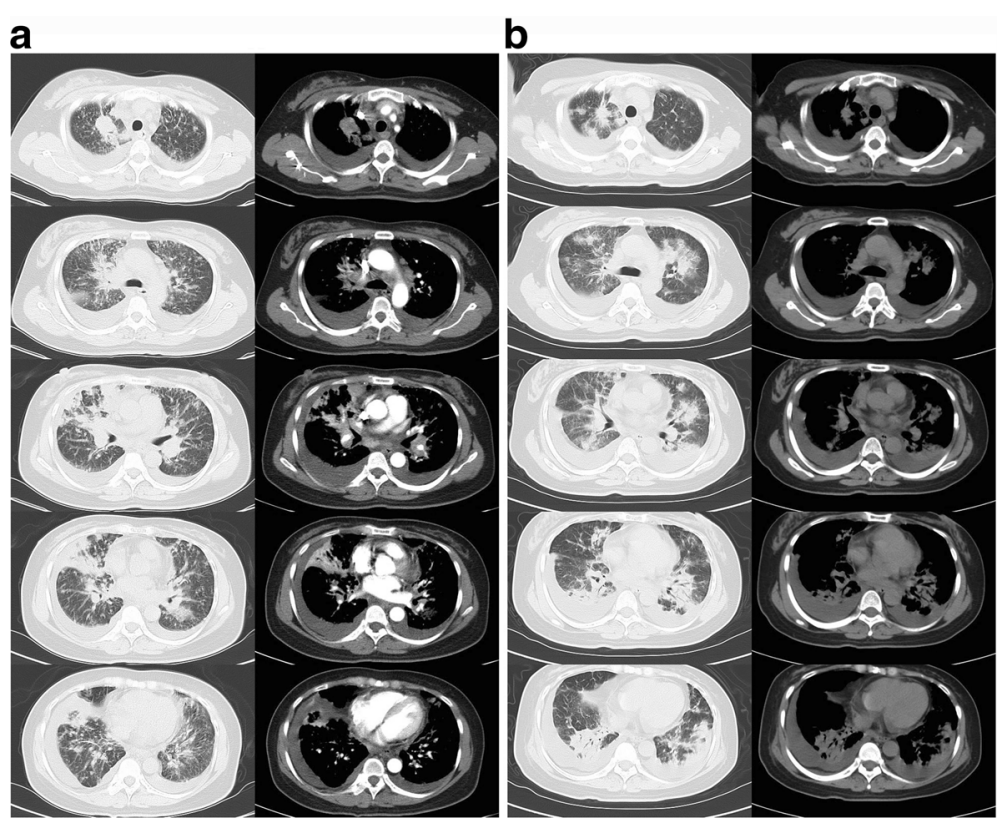

Fig. 1 a. Prior to treatment with crizotinib, a chest CT scan revealed a large irregularly shaped mass on the upper right lobe, accompanied by multiple nodules, plaques and consolidated masses of diverse sizes randomly distributed in both lung fields. Nodular thickening of the interlobular septa and fissures, which suggested lymphangitis carcinomatosa, hilar and mediastinal lymphadenopathy, and bilateral pleural effusions, were identified as well. b Ten days after the initiation of crizotinib, chest CT revealed a significant shrinkage of intrapulmonary neoplastic lesions, lymphadenitis and lymphadenectasis but not of multiple new-onset ground-glass opacities and consolidation lesions throughout both lungs 
Table 1 The rest important blood and sputum testing indicators of pre- and post-treatment with Crizotinib

\begin{tabular}{|c|c|c|c|c|c|c|c|c|}
\hline & \multicolumn{7}{|c|}{ Blood testing indicators } & \multirow{2}{*}{$\begin{array}{l}\text { Sputum } \\
\text { indicators } \\
\text { Sputum culture }\end{array}$} \\
\hline & $\begin{array}{l}\text { Carbohydrate } \\
\text { antigen } 199 \\
(\mathrm{IU} / \mathrm{ml})\end{array}$ & $\begin{array}{l}\text { Cytokeratin } \\
\text { fragment } \\
\text { (ug/L) }\end{array}$ & $\begin{array}{l}\text { Neuron-specific } \\
\text { enolase } \\
\text { (ug/L) }\end{array}$ & $\begin{array}{l}\text { Squamous cell } \\
\text { carcinoma antigen } \\
\text { (ug/L) }\end{array}$ & $\begin{array}{l}\text { White blood cell } \\
\text { count }\left(\times 10^{9} / \mathrm{L}\right)\end{array}$ & $\begin{array}{l}\text { Erythrocyte } \\
\text { sedimentation } \\
\text { rate }(\mathrm{mm} / \mathrm{h})\end{array}$ & $\begin{array}{l}\text { C-reactive } \\
\text { protein (mg/L) }\end{array}$ & \\
\hline Normal range & $0.0 \sim 37.0$ & $0.00 \sim 7.00$ & $0.00 \sim 10.00$ & $0.00 \sim 2.50$ & $3.5 \sim 9.5$ & $0 \sim 20$ & $0 \sim 8$ & NA \\
\hline Pre-treatment & 100.8 & 14.31 & 4.85 & 22.94 & 11.3 & 32 & 95.1 & negative \\
\hline $\begin{array}{l}\text { Post- } \\
\text { treatment }\end{array}$ & 57.3 & 15.14 & 5.84 & 7.89 & 16.7 & 10 & 4.1 & negative \\
\hline
\end{tabular}

In addition, a re-examination of blood tumour markers, infection-related indicators, and characteristics of hydrothorax, combined with percutaneous lung biopsy, was undertaken to clarify the cause. CEA in the pleural fluid and serum increased to $13.6 \mu \mathrm{g} / \mathrm{L}$ and $52.7 \mu \mathrm{g} / \mathrm{L}$, respectively, after crizotinib medication. Other important indicators are described in Table 1. Consistent with the CT scan, the pathology of hydrothorax suggested a significantly reduced number of tumour cells. Three columnar specimens of percutaneous biopsy from the new-onset consolidation area on the lower left pulmonary were collected promptly, all of which were $1 \mathrm{~cm}$ in length and 18 gauge in diameter. Histological observation of these biopsies revealed a diffuse alveolar oedema, thickening of the septa (Fig. 2d), macrophages or foamy macrophages prominently present in differently sized alveolar spaces (Fig. 2e), mild infiltration of inflammatory lymphocytes, monocytes, fibroblasts, and myofibroblasts in the interstitium (Fig. 2f), rare and atypical hyaline membrane formations in alveolar spaces (Fig. 2g), multiple hyaline thrombi (microthrombi) in pulmonary arterioles of partial areas (Fig. 2h), and atypical hyperplasia of type II alveolar epithelial cells in localized areas (Fig. 2i). There was no evidence of infections and

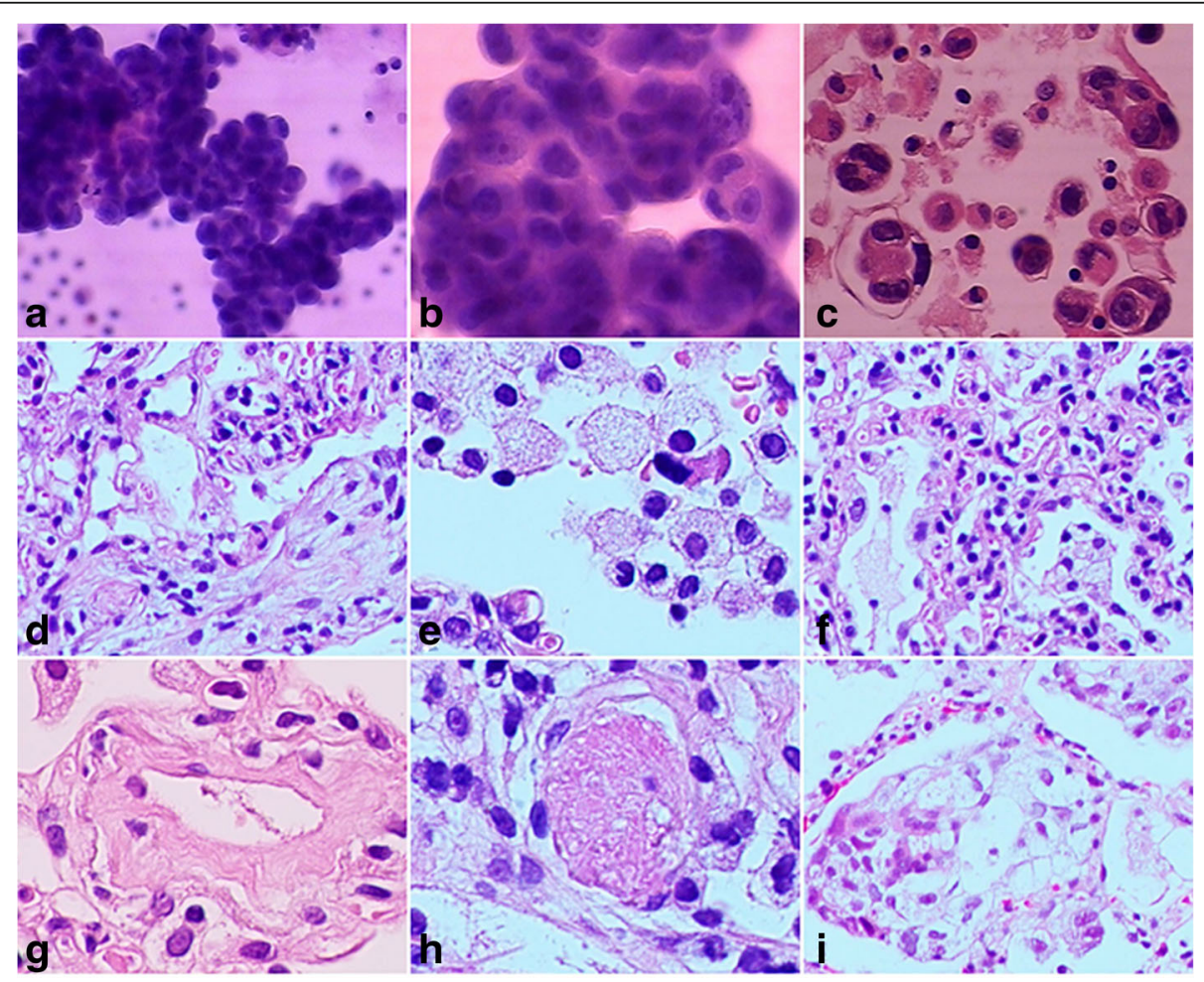

Fig. 2 a-c HE staining $(\times 200),(\times 400),(\times 200)$, liquid-based cell smear and a cell wax block revealed that tumour cells with gigantic nucleoli were distributed in clusters and presented with a round-edge, strongly encapsulated, obviously three-dimensional structure. Fig. 2d-I HE staining showed histological characteristics of acute interstitial pneumonia. Fig. $2 \mathbf{d}(\times 200)$ Diffuse alveolar oedema, thickening of the septa. Fig. 2e ( $\times 400)$ Macrophages or foamy macrophages prominently present in differently sized alveolar spaces. Fig. $2 \mathbf{f}(\times 200)$ Mild infiltration of inflammatory cells in the interstitium. Fig. $2 \mathbf{g}(\times 400)$ Rare and atypical hyaline membrane formations in alveolar spaces. Fig. $2 \mathbf{h}(\times 400)$ Multiple hyaline thrombi (microthrombi) in pulmonary arterioles of partial areas. Fig. $2 \mathbf{i}(\times 400)$ Atypical hyperplasia of type II alveolar epithelial cells in localized areas 


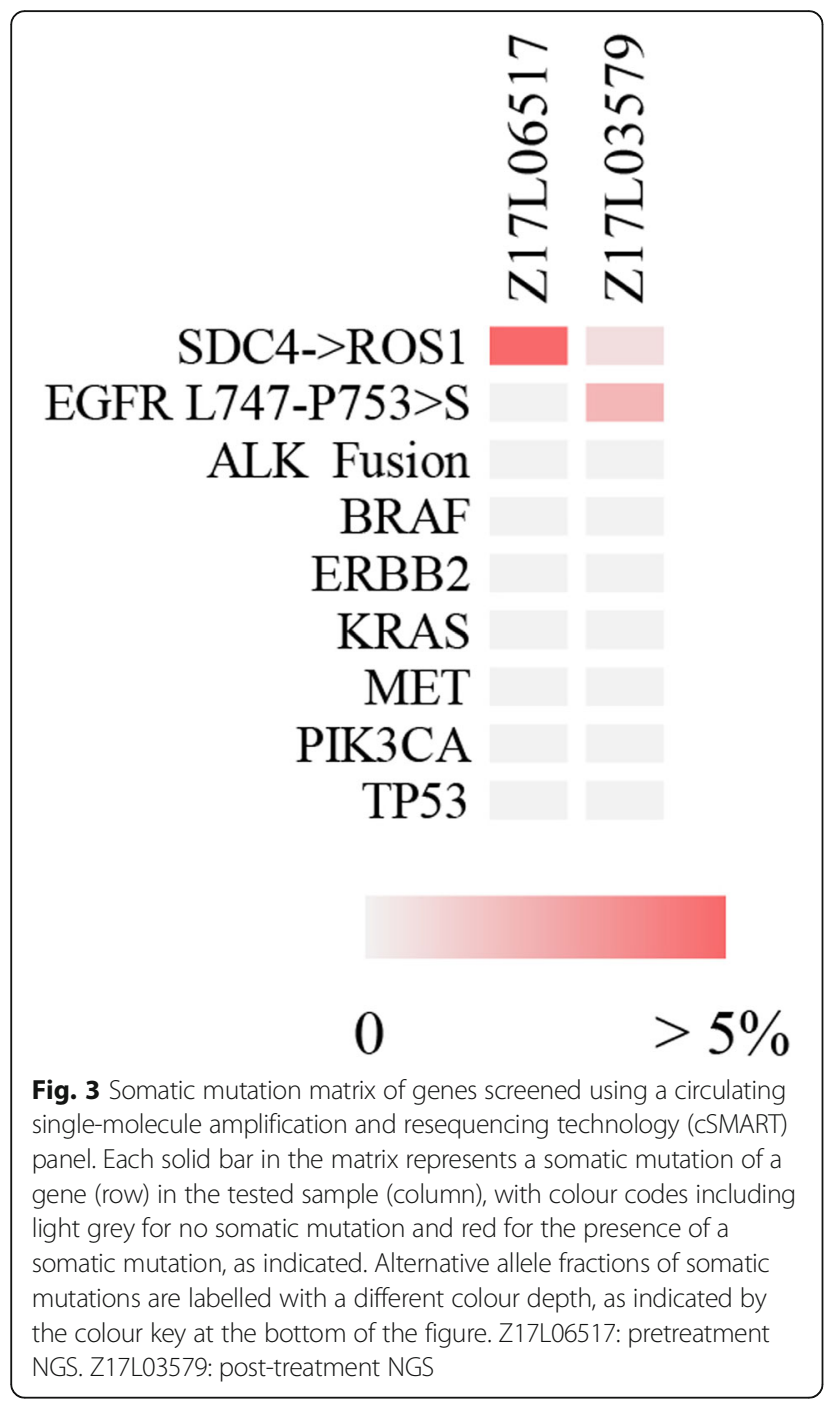

invasion of tumour cells. All these pathological changes were consistent with acute lung injury. The pathological stage was assumed to be a transition from exudation to organization. The patient denied having previous pulmonary-related diseases, and there were no other drugs that might potentially cause lung toxicity during crizotinib treatment. Therefore, we made a diagnosis of crizotinib-induced ILD.

MP pulse therapy ( $0.5 \mathrm{~g}$ once per day) was immediately substituted for the original crizotinib treatment and applied for three days. Tracheal intubation and mechanical ventilation were also undertaken because of progressive deterioration leading to respiratory failure. All necessary and additional treatment procedures were conducted to prevent ILD, but the patient died 20 days after the first administration of crizotinib. Upon approval of her family, next-generation sequencing analysis of a lung biopsy sample was performed, which revealed a de novo exon 19 deletion mutation in EGFR, not detected in MPE before treatment with crizotinib (Fig. 3 Z17L03579, Fig. 4a). On the other hand, the frequency of ROS1 rearrangement decreased after crizotinib treatment (Fig. 3). The occurrence of this phenomenon deserves special consideration and investigation of current crizotinib treatment in NSCLC patients.

\section{Ethics, consent and permissions}

Written informed consent has been obtained from the patient's family for the publication of this case report and accompanying images.

\section{Discussion}

In this study, we report the first case of fatal crizotinib-induced ILD in a ROS1-positive NSCLC patient. ROS1 rearrangements are identified in 1 to $2 \%$ of patients with NSCLC [1]. Up to now, crizotinib is still the only targeted agent approved for NSCLC patients
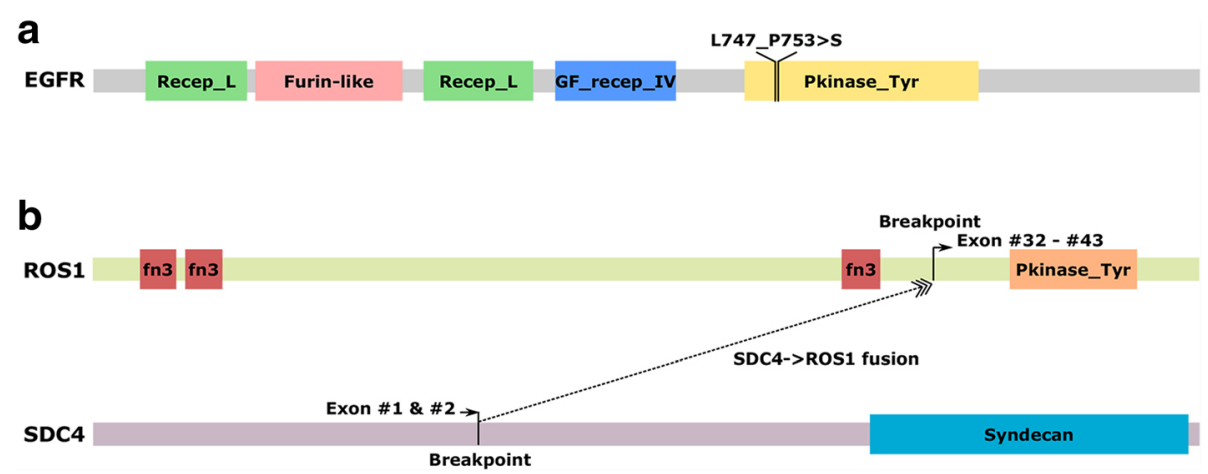

Fig. 4 Genomic organization of somatic alterations identified in our patient. a complex indel lying in EGFR exon 19, which affects the protein tyrosine kinase domain. $\mathbf{b}$ Translocation resulted in a fusion product with exons 1 and 2 of SDC4 and exons 32-43 of ROS1, including the protein tyrosine kinase domain. Abbreviations of protein domains: Recep $L$, receptor $L$ domain; Furin-like, furin-like cysteine-rich region; GF recep IV, growth factor receptor domain IV; Pkinase Tyr, protein tyrosine kinase domain; fn3, fibronectin type III domain; Syndecan, syndecan domain 
with ROS1 rearrangements. A retrospective review of 4 PROFILE clinical trials indicated that the overall incidence of crizotinib-induced ILD was $1.2 \%$, but its mortality rate was up to $50 \%$ [2]. It is a rare but serious adverse event in patients on crizotinib therapy.

Although crizotinib-induced ILD in ROS1-positive NSCLC patients has not been systematically characterized before, patients with ROS1 rearrangements had a similar incidence $(1.9 \%, 1$ of 53$)$ of ILD as those containing ALK rearrangements $(1.7 \%, 2$ of 119) in a phase I PROFILE 1001 study of crizotinib [3]. In addition, the safety profiles of crizotinib are similar for ALK-positive and ROS1-positive NSCLC patients [4]. The factors contributing to crizotinib-induced ILD in ROS1-positive patients remain unclear, whereas risk factors significantly correlated with the development of ILD in ALK-positive NSCLC patients have been described and include age, poor performance status, smoking status, past/concomitant ILD and concomitant pleural effusion [5]. In this case report, a compromised performance status, lymphangitis carcinomatosa and bilateral pleural effusions were observed in the chest and, hence, could be the key factors contributing to the development of ILD.

The median duration from the initiation of crizotinib therapy to the onset of ILD was found to be 23 days (range: 3-763 days) [2]. Créquit et al. described that a severe, usually fatal ILD developed within the 1st month of treatment, and its chest CT manifestation included an early onset of ground-glass opacity, which diffused and spread rapidly in both lungs [6]. In our case, in addition to multiple ground-glass opacity lesions, multiple consolidation lesions were found simultaneously, which coincided with our pathological staging features of the transition from exudation to the organizing period. Moreover, it was reasonable to assume that the clinical symptoms and imaging changes of interstitial pneumonia would be observed earlier than the tenth day without MP administration at the time of admission.

Possible pathological causation of crizotinib-induced ILD has been discussed in several studies [6-8]. In these studies, specimens were obtained either from bronchoscopic lung biopsy, alveolar lavage fluid or corpse biopsy. Limited by the quality and phase of biopsy specimen collection, these pathological findings could only reveal incomplete characteristics of interstitial pneumonia. To explore the pathology of the case reported in this study, we applied an HE staining technique to three freshly coloured, cylindrical percutaneous biopsy samples, which fully met the requirements for diagnosis of ILD. We first excluded the possibility of infection, and no invasion of tumour cells could be observed either. Histological characteristics, such as a thickened alveolar septa, infiltration of inflammatory cells in the interstitium, foamy macrophages and hyalinosis in alveolar cavities, hyaline thrombi (microthrombi) in pulmonary arterioles, and atypical hyperplasia of type II alveolar epithelial cells, corresponded to typical diffuse alveolar damage. The pathological stage of transition from exudation to the organizing period was also proposed. As far as we are aware, all these detailed pathological features have not been reported in the literature so far.

It is worth noting that a de novo EGFR mutation was detected in the ILD biopsy, which was not found at the time of admission. One possible reason could be a low abundance of EGFR-mutated subclones that already existed in tumour tissue at the time of admission. These cells survived the crizotinib treatment, and their abundance increased. Recently, several studies have suggested that ROS1 rearrangements co-occur with mutations in EGFR at clinically relevant frequencies [9-11]. Another reason might be that crizotinib treatment induces acquired mutations in EGFR. Activation of EGFR, which enables cancer cells to bypass crizotinib-mediated inhibition of ROS1 signalling, has been described as a mechanism of resistance to crizotinib in ROS1-rearranged NSCLC [12]. Interestingly, in ROS1-rearranged and other fusion kinase-driven cell line models, EGFR activation and signalling appear to serve as an important early adaptive survival response to TKI exposure [13]. In this case, a decrease in original tumour lesions was observed before the time of lung biopsy, suggesting that the detectable EGFR mutations had not triggered crizotinib resistance in this period. ILD can be frequently caused by targeted therapy in EGFR-positive patients; however, the link between EGFR mutations, targeted therapy and ILD requires further investigation. It is also notable that there were no tumour cells detected in the second round of pathological diagnosis in the patient. Therefore, the detection of the EGFR mutation could be due to DNA fragments of ruptured tumour cells merging with interstices. In summary, there is not sufficient evidence to demonstrate that the EGFR mutation resulted in crizotinib resistance in this case because of radiological shrinkage of original tumour lesions and pathological features of new-onset lesion biopsy. Nevertheless, it is worth investigating the relationship between EGFR mutations and ILD in the future.

The limitations of this case report are that we did not detect hydrothorax after the treatment, and other gene mutation-detecting methods were not used to further examine all the specimens to exclude detection error.

\section{Conclusions}

In conclusion, advanced non-small-cell lung cancer in patients with ROS1 rearrangements, treated with crizotinib, may be accompanied by fatal ILD in the initial period. Histopathology and molecular pathological examination of lung biopsy specimens is crucial for differential diagnosis and treatment guidance. 


\section{Abbreviations}

ALK: anaplastic lymphoma kinase; BRAF: B-Raf proto-oncogene;

CEA: carcinoembryonic antigen; CK 7: cytokeratin 7; CT: computed tomography; EGFR: epidermal growth factor receptor; FDA: Food and Drug Administration; HE: haematoxylin-eosin; HER2: human epidermal growth factor receptor 2; ILD: interstitial lung disease; KRAS: kirsten rat sarcoma virus; MP: methylprednisolone; MPE: malignant pleural effusion; NSCLC: non-smallcell lung cancer; ROS1: c-ros oncogene 1; TKI: tyrosine kinase inhibitor; TTF1: thyroid transcription factor-1

\section{Availability of data and materials}

All data generated or analysed during this study are included in this published article.

\section{Authors' contributions}

SBW and KTL conceived and designed the study. FR, DP and FR performed the analyses. SBW and DP prepared all tables. KTL and DWZ wrote the main manuscript. All authors reviewed the manuscript. All authors read and approved the final manuscript.

\section{Ethics approval and consent to participate}

The study was performed in accordance with the Declaration of Helsinki and was approved by the Lihuili Hospital Cancer Centre Ethics Review Board.

\section{Consent for publication}

Written informed consent has been obtained from the patient's family for the publication of this case report and accompanying images.

\section{Competing interests}

The authors declare that they have no competing interests.

\section{Publisher's Note}

Springer Nature remains neutral with regard to jurisdictional claims in published maps and institutional affiliations.

\begin{abstract}
Author details
'Department of Respiratory Medicine, Lihuili Hospital, Ningbo Medical Center, No. 57, Xin'ning Road, Ningbo 315041, China. ${ }^{2}$ Department of Radiation Oncology, Lihuili Hospital, Ningbo Medical Center, Ningbo 315041, China. ${ }^{3}$ Department of Radiology, Lihuili Hospital, Ningbo Medical Center, Ningbo 315041, China. ${ }^{4}$ Department of Thoracic Surgery, Lihuili Hospital, Ningbo Medical Center, Ningbo 315041, China. ${ }^{5}$ Department of Diagnosis, Ningbo Diagnostic Pathology Center, No. 79, Huan'cheng Road, Ningbo 315021, China
\end{abstract}

Received: 24 April 2018 Accepted: 2 July 2018

Published online: 20 July 2018

\section{References}

1. Bergethon K, Shaw AT, Ou SH, et al. ROS1 rearrangements define a unique molecular class of lung cancers. J Clin Oncol. 2012;30:863-70.

2. Yoneda KY, Scranton JR, Michael A, et al. Interstitial lung disease associated with Crizotinib in patients with advanced none small cell lung Cancer: independent review of four PROFILE trials. Clin Lung Cancer. 2015;18(5):472-9.

3. Data on file (62a). Pfizer.

4. Shaw AT, Ou S-HI, Bang Y-J, et al. Crizotinib in ROS1-rearranged non-smallcell lung Cancer. N Engl J Med. 2014;371:1963-71.

5. Gemma A, Kusumoto M, Kurihara Y, et al. Analysis of Data on Interstitial Lung Disease Onset and Its Risk Following Treatment of ALK-positive NSCLC with Xalkori [poster]. Presented at WCLC 2017, October 15-18; Yokohama, Japan. Abstract no. 9146

6. Créquit $P$, Wislez M, Fleury Feith J, et al. Crizotinib associated with groundglass opacity predominant pattern interstitial lung disease. J Thorac Oncol. 2015;10:1148-55.

7. Tamiya A, Okamoto S, Miyazaki M, Shimizu S, Kitaichi M, Nakagawa K. Severe acute interstitial lung disease after Crizotinib therapy in a patient with EML4-ALKepositive none-small-cell lung cancer. J Clin Oncol. 2013;31:149-51.
8. Tachihara M, Kobayashi K, Ishikawa Y, et al. Successful Crizotinib Rechallenge after Crizotinib-induced interstitial lung disease. Jpn J Clin Oncol. 2014;44(8):762-4.

9. Rimkunas VM, Crosby KE, Li D, et al. Analysis of receptor tyrosine kinase ROS1-positive tumors in non-small cell lung cancer: identification of a FIG-ROS1 fusion. Clin Cancer Res. 2012;18:4449-57.

10. Wiesweg M, Eberhardt WE, Reis $\mathrm{H}$, et al. High prevalence of concomitant oncogene mutations in prospectively identified patients with ROS1-positive metastatic lung cancer. J Thorac Oncol. 2017;12:54-64.

11. Lin JJ, Ritterhouse LL, Ali SM, et al. ROS1 fusions rarely overlap with other oncogenic drivers in non-small cell lung cancer. J Thorac Oncol. 2017;12:872-7.

12. Davies KD, Mahale S, Astling DP, et al. Resistance to ROS1 inhibition mediated by EGFR pathway activation in non-small cell lung cancer. PLoS One. 2013:8(12):e82236.

13. Vaishnavi A, Schubert L, Rix U, et al. EGFR mediates responses to smallmolecule drugs targeting oncogenic fusion kinases. Cancer Res. 2017;77:3351-63.

\section{Ready to submit your research? Choose BMC and benefit from:}

- fast, convenient online submission

- thorough peer review by experienced researchers in your field

- rapid publication on acceptance

- support for research data, including large and complex data types

- gold Open Access which fosters wider collaboration and increased citations

- maximum visibility for your research: over $100 \mathrm{M}$ website views per year

At BMC, research is always in progress.

Learn more biomedcentral.com/submissions 